Vol. 1, No. 1, 2019

https://doi.org/10.23939/cds2019.01.012

УДК 519.2.4; 681.5; 621.382

A. Holovatyy, V. Teslyuk, K. Kolesnyk, V. Tabala

Lviv Polytechnic National University

\title{
DEVELOPMENT OF DIGITAL COMPASS BASED ON AVR MICROCONTROLLER AND MEMS ACCELEROMETER- MAGNETOMETER MODULE LSM303DLHC
}

\author{
(C) Holovatyy A., Teslyuk V., Kolesnyk K., Tabala V., 2019
}

In the paper, the digital compass based on AVR microcontroller has been developed using MEMS accelerometer-magnetometer LSM303DLHC. The structure and hardware of the digital compass have been developed. The calibration algorithms of the accelerometer and magnetometer of the LSM303DLHC module have been described. The calculation algorithm of the pitch and roll angles has been described. The heading calculation algorithm of the digital compass without tilt compensation and with tilt compensation has been described. The operation algorithm and embedded software of the digital compass have been developed. The model and prototype of the digital compass have been created. The simulation and operation test of the developed digital compass have been performed.

Key words: digital compass, Arduino Uno R3, AVR microcontroller ATmega328P-PU, accelerometer, magnetometer, MEMS module LSM303DLHC, Proteus Design Suite, C programming language, Arduino IDE, embedded software.

\section{Introduction}

The development of electronics and micro-electro-mechanical systems technology has led to the appearance of integral motion sensors, such as accelerometers, gyroscopes, magnetometers etc. Magnetometer is a device for measuring the magnetic field strength (or intensity of one and more magnetic field components). MEMS magnetometers are widely used in handheld electronic devices, such as: smartphones, tablets, cars, robotics, etc. They are mostly a part of complex navigation systems, and in combination with an accelerometer and/or gyroscope represent an inertial navigation system that can accurately determine a location in three-dimensional space. Nowadays, the market has a wide choice of two- and three-axis integrated electronic compasses and modules with digital interfaces that contain a three-axis accelerometer and three-axis magnetometer [1,2]. The aim of the work is to develop the tiltcompensated digital compass based on AVR microcontroller and using MEMS module (accelerometermagnetometer) LSM303DLHC and 128×64 graphic LCD ST7920.

\section{Determination of the Earth's magnetic field strength and compass operation principle}

For a more complete understanding of the compass operation principle, consider the basics of the magnetism theory and the principles for determination of the Earth's magnetic field vector direction (heading or azimuth).

The magnetic field strength of the Earth is from 0.5 to 0.6 Gauss. The Earth's magnetic field vector is parallel to its surface. It always orientates to the north magnetic pole. In the northern hemisphere, this field is directed downwards. At the equator, it is directed horizontally and in the southern hemisphere it is directed upwards [3, 4]. 


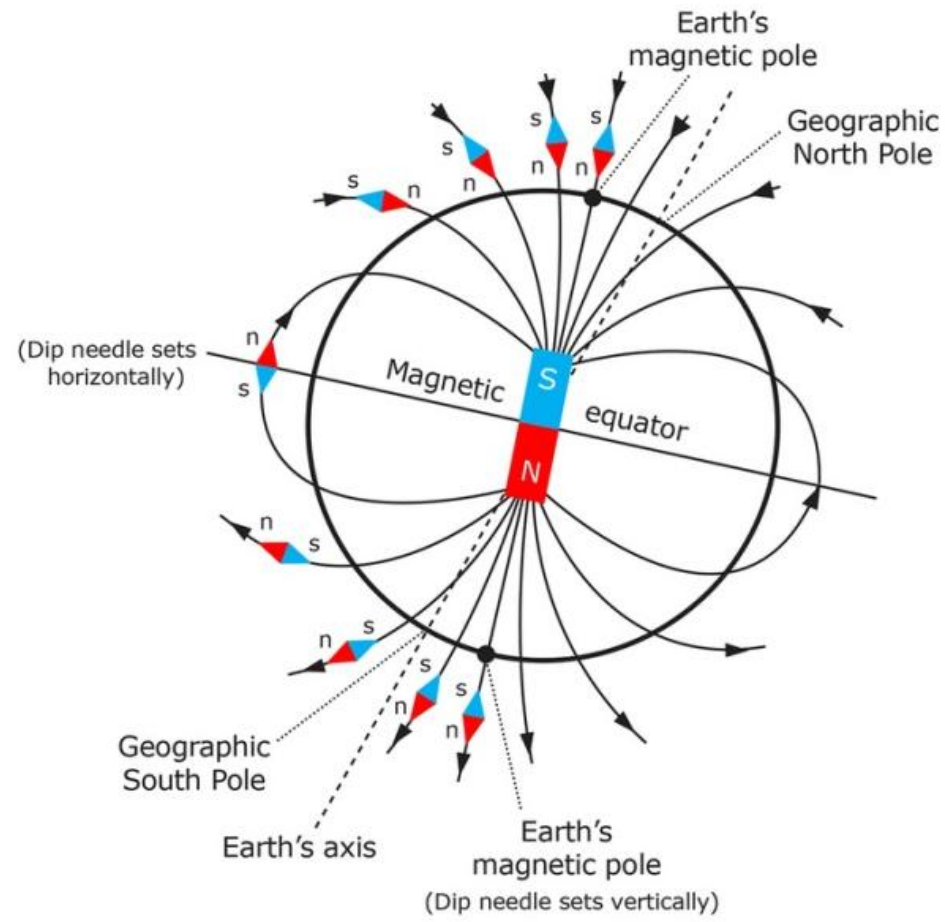

Fig. 1. Earth's magnetic field

For the creation of the tilt-compensated electronic compass, a 3-axis magnetic sensor (magnetometer) and a 3-axis accelerometer sensor (accelerometer) are needed. The accelerometer is used to measure the pitch and roll angles for the tilt compensation.

The magnetic sensor is used to measure the Earth's magnetic field strength and determine the heading angle relative to the magnetic north pole. $[5,6]$.

Terminology

In the compass the aviation terminology is used to determine the coordinates of the device and the three angles: pitch, roll and heading as shown in Fig. 2.

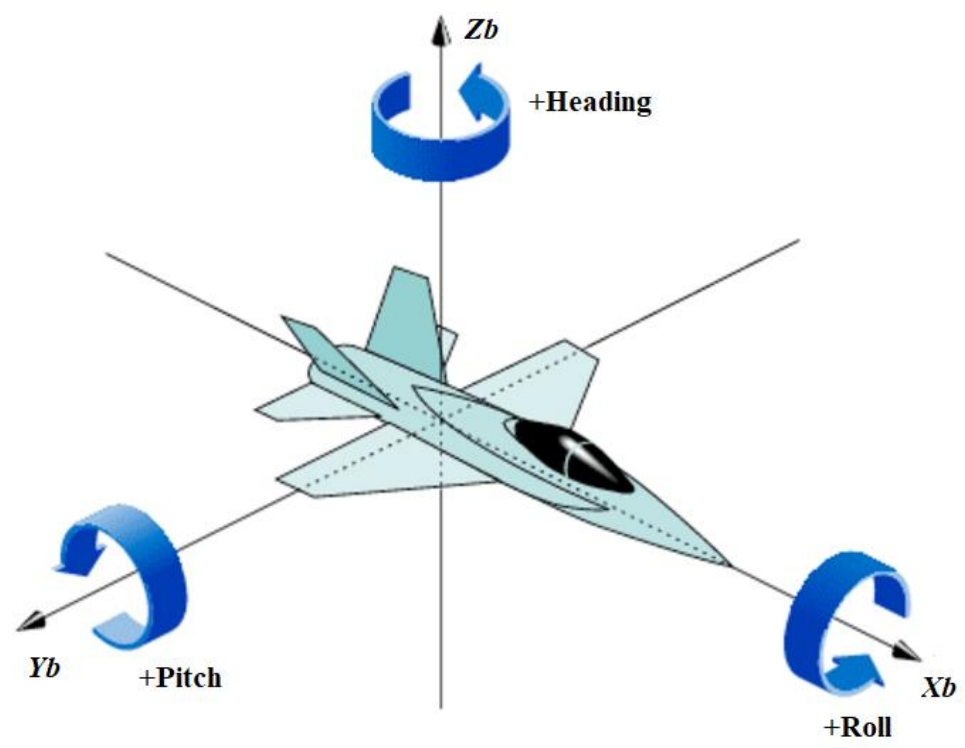

Fig. 2. Body coordinates and attitude angles 
In Fig. 2, coordinates of the device $X_{b}, Y_{b}$ and $Z_{b}$ are directed forward, right, down based on the rule of the right hand. Three attitude angles (pitch, roll and heading) are referenced to the local horizontal plane that is perpendicular to the earth's gravity.

- Heading or azimuth $(\varphi)$ is the angle between the Xb-axis and the north magnetic pole on the horizontal plane. It is measured in a clockwise direction from the top view of the device.

- Pitch $(\rho)$ is the angle between the $X_{b}$-axis and the horizontal plane. When the device is rotated around the $Y_{b}$-axis, the axis moves upward, is positive and increases.

- Roll $(\gamma)$ is the angle between the $Y_{b}$-axis and the horizontal plane. When the device is rotated around the $X_{b}$-axis, the $Y_{b}$-axis moves downwards. Roll is positive and increases.

\section{Compass heading calculation}

When the device is at an aligned position, pitch and roll angles are equal to $0^{\circ}$. Then, the angle can be determined directly as shown in Fig. 3.

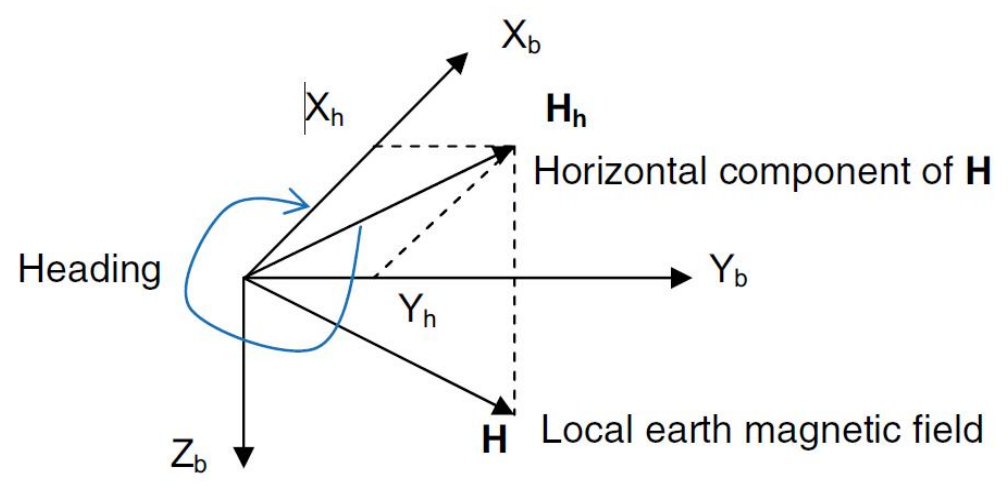

Fig. 3. Heading (azimuth) calculation

The Earth's magnetic field $(\mathrm{H})$ has a horizontal component $\mathrm{H}_{\mathrm{h}}$ which points to the north magnetic pole of the Earth. In practice, for the determination of the heading (vector direction) of the Earth's magnetic field $(\mathrm{H})$ the strength of its two components $X_{h}$ and $Y_{h}$ must be measured and angle between them has to be calculated (Fig. 3).

These components can be measured by the magnetometer. Then, the heading angle $(\varphi)$ is calculated by the following formula:

$$
\phi=\arctan \left(\frac{Y_{h}}{X_{h}}\right)
$$

In Fig. 2, when the device body $X_{b}$-axis is parallel to $\mathrm{H}_{\mathrm{h}}$ that indicates the north magnetic pole, then $X_{h}$ is max and $Y_{h}$ is 0 , so the heading $(\varphi)$ equals to $0^{\circ}$. The clockwise rotation of the device on the horizontal plane leads to the increase of the heading. When $X_{h}=0$ and $Y_{h}=$ min, then heading angle $\varphi=90^{\circ}$. Continuing the rotation to $X_{h}=\min$ and $Y_{h}=0$, then heading $=180^{\circ}$. After a $360^{\circ}$ rotation, the user will see a centered circle if the values of $X_{h}$ and $Y_{h}$ are obtained from the measurements of the magnetic sensor.

\section{Tilt compensation}

If the handheld device is tilted, the angles of the pitch and roll are not equal to $0^{\circ}$, as shown in Fig. 4. The pitch and roll can be measured by a three-axis accelerometer. Therefore, the values measured by the magnetic sensor $X_{M}, Y_{M}$, and $Z_{M}$ must be offset to get $X_{h}$ and $Y_{h}$, as shown in the eq. 2 . And then the eq. 1 is applied to calculate the heading. 


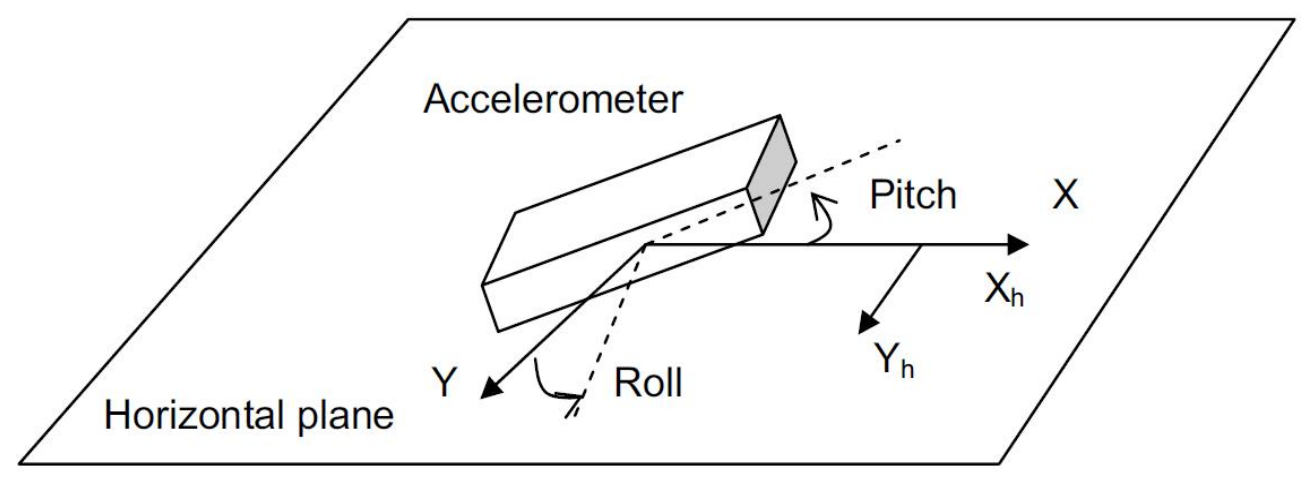

Fig. 4. Handheld device at tilted position

$$
\begin{gathered}
X_{h}=X_{M} \cos (\rho)+Z_{M} \sin (\rho) \\
Y_{h}=X_{M} \sin (\gamma) \sin (\rho)+Y_{M} \cos (\gamma)-Z_{M} \sin (\gamma) \cos (\rho),
\end{gathered}
$$

where $X_{M}, Y_{M}$ and $Z_{M}$ - values measured by the magnetometer.

\section{LSM303DLHC accelerometer calibration algorithm}

All STMicroelectronics MEMS accelerometers are calibrated that is sufficient for the most applications. For getting the heading accuracy less than $2^{\circ}$, the following calibration procedure is required. After the module installation into a handheld device, it must be re-calibrated. The accelerometer is recalibrated for determination of the bias value and scale factor, and the error matrix relative to the device axes $X_{b}, Y_{b}$ and $Z_{b}$. The ratio between the normalized values $A_{x l}, A_{y l}$, and $A_{z l}$ and the measured accelerometer values $A_{x}, A_{y}$, and $A_{z}$ is determined as:

$$
\begin{aligned}
& {\left[\begin{array}{l}
A_{x 1} \\
A_{y 1} \\
A_{z 1}
\end{array}\right]=\left[A_{-} m\right]_{3 \times 3}\left[\begin{array}{ccc}
1 / A_{-} S C_{x} & 0 & 0 \\
0 & 1 / A_{-} S C_{y} & 0 \\
0 & 0 & 1 / A_{-} S C_{z}
\end{array}\right] \cdot\left[\begin{array}{l}
A_{x}-A_{-} O S_{x} \\
A_{y}-A_{-} O S_{y} \\
A_{z}-A_{-} O S_{z}
\end{array}\right]=} \\
&= {\left[\begin{array}{ccc}
A C C_{11} & A C C_{12} & A C C_{13} \\
A C C_{21} & A C C_{22} & A C C_{23} \\
A C C_{31} & A C C_{32} & A C C_{33}
\end{array}\right] \cdot\left[\begin{array}{l}
A_{x} \\
A_{y} \\
A_{z}
\end{array}\right]+\left[\begin{array}{l}
A C C_{10} \\
A C C_{20} \\
A C C_{30}
\end{array}\right], }
\end{aligned}
$$

where $\left[A \_m\right]-3 \times 3$ misalignment matrix between the accelerometer sense axes and the device axes; $A \_S C i$ $(\mathrm{i}=x, y, z)$ is the scale factor and A_OSi is the offset.

The accelerometer calibration is needed for determination of the 12 parameters from $A C C_{10}$ to $A C C_{33}$. So that with any given raw data arbitrary positions, normalized values can be obtained $[7,8]$.

Calibration can be done at the 6 stationary positions. The accelerometer data in each position known as $A_{x l}, A_{y l}$, and $A_{z l}$ should be collected during 5-10 sec. Then, the method of least squares can be used to get the 12 optimal calibration parameters for the accelerometer.

\section{LSM303DLHC magnetometer calibration algorithm}

The LSM303DLHC magnetometer has the measurement resolution of the magnetic field $8 \mathrm{mG}$ (milligauss) at VDD $=+3 \mathrm{~V}$. The average magnitude of the horizontal component of the magnetic field is approximately in the range of $200 \mathrm{mG}$ (more on the equator, less closer to the magnetic poles). Therefore, the expected compass heading accuracy is approximately $2.3^{\circ}[=\operatorname{arctg}(8 / 200)]$. Within the range \pm 1.3 gauss, the LSM303DLHC magnetic sensor sensitivity is $1055 \mathrm{LSB} /$ gauss for the $\mathrm{X} / \mathrm{Y}$ axes and $950 \mathrm{LSB}$ /gauss for the Z-axis. 
The relationship between the normalized $M_{x l}, M_{y l}$, and $M_{z l}$ and the measured values of the magnetometer can be expressed by the following equation:

Equation 4:

$$
\begin{aligned}
& {\left[\begin{array}{c}
M_{x 1} \\
M_{y 1} \\
M_{z 1}
\end{array}\right]=\left[M_{-} m\right]_{3 \times 3}\left[\begin{array}{ccc}
1 / M_{-} S C_{x} & 0 & 0 \\
0 & 1 / M_{-} S C_{y} & 0 \\
0 & 0 & 1 / M_{-} S C_{z}
\end{array}\right] \times} \\
& \times\left[M_{-} s i\right]_{3 \times 3}\left[\begin{array}{c}
M_{x}-M_{-} O S_{x} \\
M_{y}-M_{-} O S_{y} \\
M_{z}-M_{-} O S_{z}
\end{array}\right]=\left[\begin{array}{ccc}
M R_{11} & M R_{12} & M R_{13} \\
M R_{21} & M R_{22} & M R_{23} \\
M R_{31} & M R_{32} & M R_{33}
\end{array}\right] \cdot\left[\begin{array}{c}
M_{x}-M R_{10} \\
M_{y}-M R_{20} \\
M_{z}-M R_{30}
\end{array}\right],
\end{aligned}
$$

where $\left[M \_m\right]$ is a $3 \times 3$ misalignment matrix between the magnetic sensor axes and the device axes; $M \_S C i$ $(\mathrm{i}=x, y, z)$ is the scale factor and $M_{-} O S i$ is the offset caused by hard-iron distortion; $\left[M_{-} s i\right]-3 \times 3$ matrix caused by soft-iron distortion.

The magnetometer calibration is used for determination of the parameters from $M R_{10}$ to $M R_{33}$, thus with any known measured values at arbitrary positions. Thus, the normalized values can be calculated for any raw measurements at arbitrary positions [7, 8].

\section{Pitch and roll calculation algorithm}

Let's suppose that the LSM303DLHC chip is installed on the device, as shown in Fig. 5.

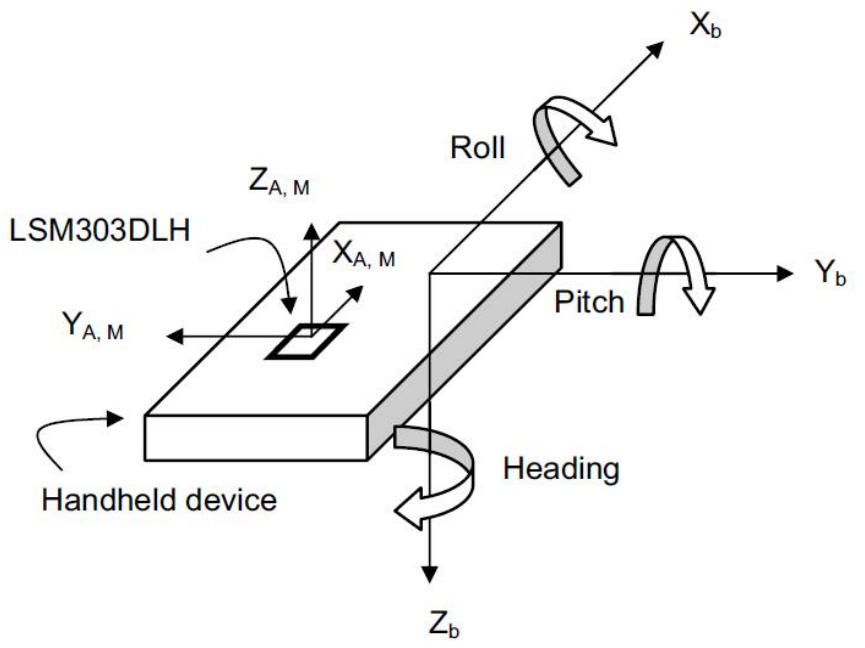

Fig. 5. Coordinate system

of the electronic compass

$X_{b}, Y_{b}$ and $Z_{b}$ are the device body axes which are directed forward, right and down, respectively. The accelerometer and magnetometer sense axes are $X_{A, M}, Y_{A, M}$, and $Z_{A, M}$, respectively. The sign of $Y_{A, M}$ and $Z_{A, M}$ from the sensor measurements has to be reversed to make the same direction of the sense axes as the axes of the device. Pitch/roll/heading angles are referenced to the local horizontal plane which is perpendicular to the Earth's gravity.

When the device is at the $3 \mathrm{D}$ arbitrary position $X^{\prime}{ }_{b}, Y^{\prime}{ }_{b}$ and $Z_{b}{ }_{b}$, there are several procedures for the device rotation from the local coordinate system $X_{b} . Y_{b}$ and $Z_{b}$, as shown in Fig. 8. The different rotation procedures lead to the different rotation matrix.

Firstly, the device has to be rotated around the $Z_{b}$-axis clockwise at the angle $(\varphi)$ with a view from the origin to downwards. Then, turn the device around the $Y_{b}$-axis at the angle $(\rho)$, the $X_{b}$-axis moves to the top. Then, the device is rotated around $X_{b}$ to the angle $(\gamma), Y_{b}$ moves to the bottom. The new coordinates of the device will be $X_{b}{ }_{b}, Y_{b}{ }_{b}$ and $Z_{b}{ }_{b}$, as shown in Fig. 5. 


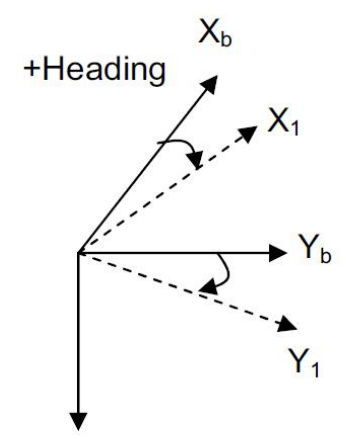

$Z_{b}, Z_{1}$

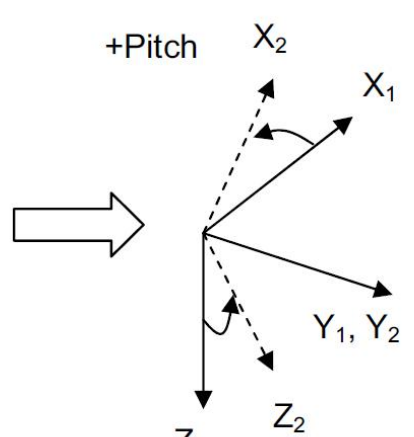

$Z_{1}$

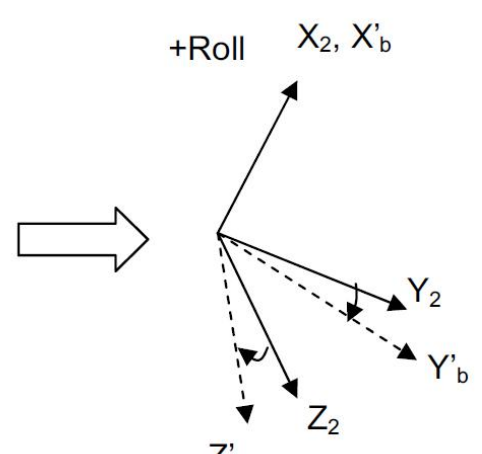

$Z_{b}^{\prime}$

Fig. 6. Rotation procedure

Then, each matrix of rotation is:

$$
\begin{aligned}
& R_{\psi}=\left[\begin{array}{ccc}
\cos \phi & \sin \phi & 0 \\
-\sin \phi & \cos \phi & 0 \\
0 & 0 & 1
\end{array}\right], \\
& R_{\rho}=\left[\begin{array}{ccc}
\cos \rho & 0 & -\sin \rho \\
0 & 1 & 0 \\
\sin \rho & 0 & \cos \rho
\end{array}\right], \\
& R_{\gamma}=\left[\begin{array}{ccc}
1 & 0 & 0 \\
0 & \cos \gamma & \sin \gamma \\
0 & -\sin \gamma & \cos \gamma
\end{array}\right] .
\end{aligned}
$$

The relationship between $X_{b}^{\prime} / Y_{b}^{\prime} / Z_{b}{ }_{b}$ and $X_{b} / Y_{b} / Z_{b}$ is the following:

$$
\left[\begin{array}{c}
X_{b}^{\prime} \\
Y_{b}^{\prime} \\
Z_{b}^{\prime}
\end{array}\right]=R_{\gamma} R_{\rho} R_{\psi}\left[\begin{array}{c}
X_{b} \\
Y_{b} \\
Z_{b}
\end{array}\right]=
$$

$$
=\left[\begin{array}{ccc}
\cos \rho \cos \phi & \cos \rho \sin \phi & -\sin \rho \\
\cos \phi \sin \rho \sin \gamma-\cos \gamma \sin \phi & \cos \gamma \cos \phi+\sin \rho \sin \gamma \sin \phi & \cos \rho \sin \gamma \\
\cos \phi \sin \rho \cos \gamma+\sin \gamma \sin \phi & -\sin \gamma \cos \phi+\sin \rho \cos \gamma \sin \phi & \cos \rho \cos \gamma
\end{array}\right] \cdot\left[\begin{array}{c}
X_{b} \\
Y_{b} \\
Z_{b}
\end{array}\right] \text {. }
$$

In the local horizontal plane (Fig. 5) $X_{b}=Y_{b}=0, Z_{b}=+1$ g. At $X_{b}{ }_{b} / Y^{\prime}{ }_{b} / Z^{\prime}{ }_{b}$, the accelerometer raw measurements are $A_{x}, A_{y}$ and $A_{z}$. They are signed integer in terms of LSBs. Let $A_{x l}, A_{y l}$ and $A_{z l}$ be the normalized values after using the accelerometer calibration parameters for $A_{x}, A_{y}$, and $A_{z}$. So $A_{x l}, A_{y l}$ and $A_{z l}$ become the floating point values less than 1 in terms of $\mathrm{g}$ and the root of the sum of their squared values should be equal to 1 when the accelerometer is still. Then the equation 9 will be:

$$
\left[\begin{array}{l}
A_{x 1} \\
A_{y 1} \\
A_{z 1}
\end{array}\right]=\left[\begin{array}{ccc}
\cos \rho \cos \psi & \cos \rho \sin \phi & -\sin \rho \\
\cos \phi \sin \rho \sin \gamma-\cos \gamma \sin \phi & \cos \gamma \cos \phi+\sin \rho \sin \gamma \sin \phi & \cos \rho \sin \phi \\
\cos \phi \sin \rho \cos \gamma+\sin \gamma \sin \phi & -\sin \gamma \cos \phi+\sin \rho \cos \gamma \sin \phi & \cos \rho \cos \phi
\end{array}\right] \cdot\left[\begin{array}{l}
0 \\
0 \\
1
\end{array}\right] .
$$

Thus, pitch and roll angles can be calculated by the following equations:

$$
\text { Pitch: } \rho=\arcsin \left(-A_{x 1}\right) \text {, }
$$




$$
\text { Roll: } \gamma=\arcsin \left(A_{y 1} / \cos \rho\right) \text {. }
$$

When pitch $= \pm 90^{\circ}$, roll has to be set to $0^{\circ}$ for avoiding singularity. The function of arcsin has a good linearity from $-45^{\circ}$ to $+45^{\circ}$, so the pitch and roll calculation accuracy degrades if tilt angles exceed this range.

The normalized value of the accelerometer $A_{z l}$ is not used for the pitch and roll calculation, but it can be used to check the equality of the magnitude $|A|=\sqrt{A_{x 1}^{2}+A_{y 1}^{2}+A_{z 1}^{2}}$ to 1 . If it does not equal to 1 , then there is the detected linear acceleration or angular acceleration.

\section{Heading calculation algorithm}

For the heading calculation, the values measured by the 3-axis magnetometer should be normalized using the calibration parameters of the magnetometer and reflected on the horizontal plane for tilt compensation.

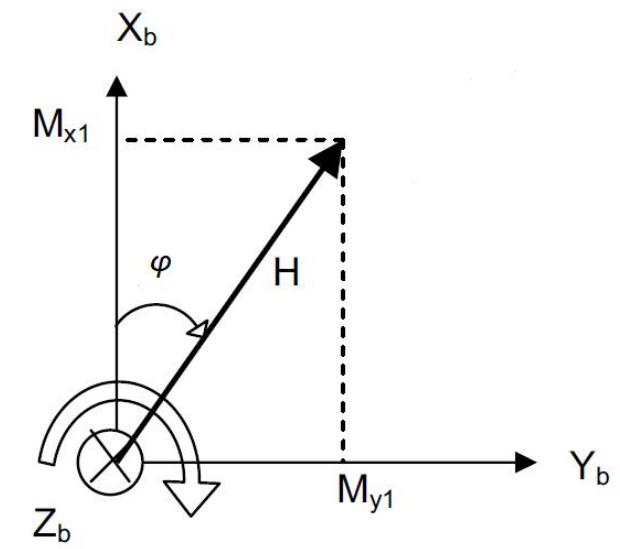

Fig. 6. Heading calculation

where: $H$ - horizontal component of the Earth's

magnetic field and $\mathrm{Zb}$ points into the page

If the device rotates from $X_{b} / Y_{b} / Z_{b}$ to $X_{b}^{\prime \prime} / Y^{\prime \prime}{ }_{b} / Z^{\prime \prime}{ }_{b}$ by roll angle rotation followed by pitch angle rotation, then:

$$
\left[\begin{array}{c}
X_{b} \\
Y_{b} \\
Z_{b}
\end{array}\right]=R_{\gamma}^{-1} R_{\rho}^{-1}\left[\begin{array}{l}
X_{b}^{\prime \prime} \\
Y_{b}^{\prime \prime} \\
Z_{b}^{\prime \prime}
\end{array}\right]=\left[\begin{array}{ccc}
\cos \rho & 0 & \sin \rho \\
\sin \gamma \sin \rho & \cos \gamma & -\sin \gamma \cos \rho \\
-\cos \gamma \sin \rho & \sin \gamma & \cos \gamma \cos \rho
\end{array}\right] \cdot\left[\begin{array}{c}
X_{b}^{\prime \prime} \\
Y_{b}^{\prime \prime} \\
Z_{b}^{\prime \prime}
\end{array}\right] .
$$

Applying the calibration parameters correction into magnetic sensor raw measurements $M_{x}, M_{y}$, and $M_{z}$ at new positions $X^{\prime \prime}{ }_{b} / Y^{\prime}{ }_{b} / Z{ }^{\prime}{ }_{b}$, the normalized magnetic sensor measurements $M_{x l}, M_{y l}$ and $M_{z l}$ will be obtained.

The values of $M_{x}, M_{y}$ and $M_{z}$ are signed integers in the terms of LSBs. The floating point values $M_{x l}, M_{y l}$ and $M_{z l}$ are less than 1 in the terms of the magnetic field strength. The square root of the sum squared values has to be equal to 1 when there is no external interference magnetic field. Thus, using the eq. 12 the tilt compensated magnetic sensor measurements $M_{x 2}, M_{y 2}, M_{z 2}$ are obtained:

$M_{x}, M_{y}$ and $M_{z}$ which are signed integer in terms of LSBs. $M_{x l}, M_{y l}$ and $M_{z l}$ are floating point values less than 1 in terms of the magnetic field strength. The square root of the sum squared values 
should be equal to 1 when the there is no external interference magnetic field. Then from the eq. 12, tilt-compensated magnetic sensor measurements $M_{x 2}, M_{y 2}$, and $M_{z 2}$ can be obtained as

$$
\begin{gathered}
M_{x 2}=M_{x 1} \cos \rho+M_{z 1} \sin \rho, \\
M_{y 2}=M_{x 1} \sin \gamma \sin \rho+M_{y 1} \cos \gamma-M_{z 1} \sin \gamma \cos \rho, \\
M_{z 2}=-M_{x 1} \cos \gamma \sin \rho+M_{y 1} \sin \gamma+M_{z 1} \cos \gamma \cos \rho .
\end{gathered}
$$

Equation 14:

$$
\begin{gathered}
\phi=\arctan \left(M_{y 2} / M_{x 2}\right) \text { for } M_{x 2}>0 \text { and } M_{y 2} \geq 0= \\
=180^{\circ}+\arctan \left(M_{y 2} / M_{x 2}\right) \text { for } M_{x 2}<0= \\
=360^{\circ}+\arctan \left(M_{y 2} / M_{x 2}\right) \text { for } M_{x 2}>0 \text { i } M_{y 2} \leq 0= \\
=90^{\circ} \text { for } M_{x 2}=0 \text { and } M_{y 2}<0 \text { or }=270^{\circ} \text { for } M_{x 2}=0 \text { and } M_{y 2}>0 .
\end{gathered}
$$

The magnitude $|M|=\sqrt{M_{x 2}^{2}+M_{y 2}^{2}+M_{z 2}^{2}}$ has to be also equal to 1 . If not, it means that an external interference magnetic field is detected.

\section{Development of digital compass}

In Fig. 7, the block diagram of the digital compass is shown. The microcontroller reads the data from the 3-axis accelerometer for the pitch and roll angle calculation, and from the 3-axis magnetometer for the heading calculation.

The algorithm of the digital compass development consists of:

- Hardware design for accelerometer and magnetometer data acquisition;

- Accelerometer calibration for obtaining the parameters needed to convert the raw data from the accelerometer to normalized values for pitch and roll angle calculation;

- Magnetometer calibration for obtaining the parameters to convert the raw data from the magnetometer to the normalized values for the heading calculation;

- Digital compass testing.

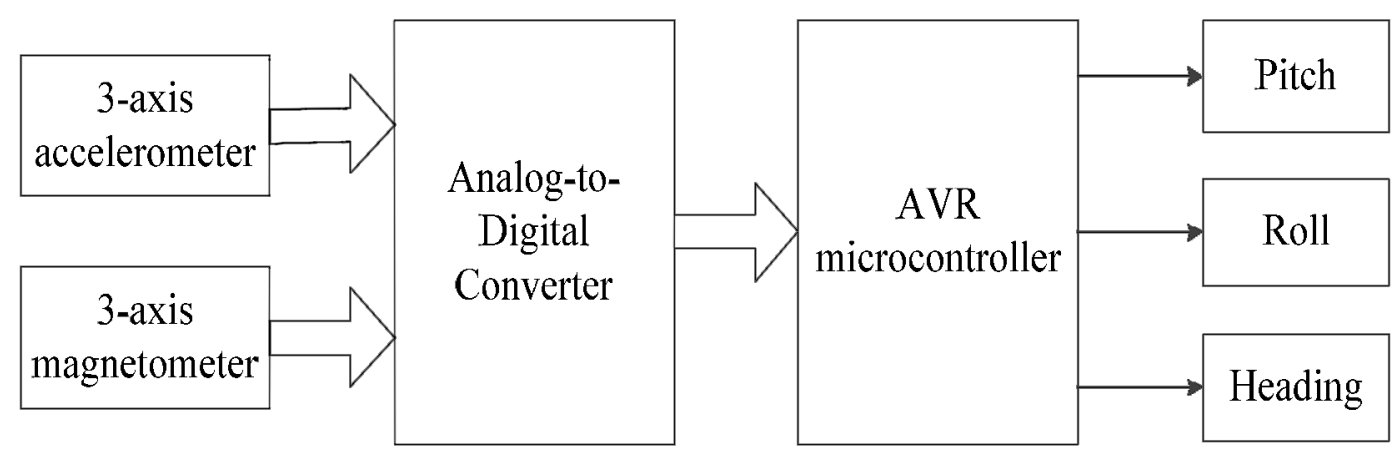

Fig. 7. Block diagram of the digital compass built

on the accelerometer-magnetometer LSM303DLHC

LSM303DLHC is a sensor module manufactured by STMicroelectronics that combines 3-axis accelerometer and 3-axis magnetometer, Fig. 8. 

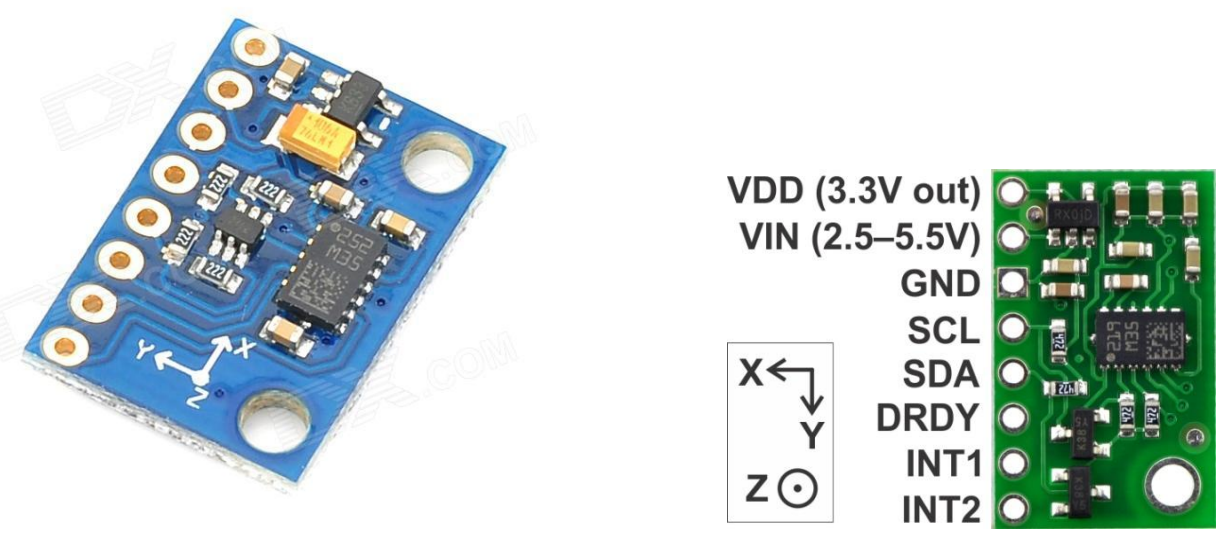

Fig. 8. The board with LSM303DLHC module and voltage regulator

LSM303DLHC can be connected to the microcontroller through the $\mathrm{I}^{2} \mathrm{C}$ digital interface. In Fig. 9, the typical LSM303DLHC connection circuit to the microcontroller via the $\mathrm{I}^{2} \mathrm{C}$ interface is shown. The power supply for the LSM303DLHC operation is $+3 \mathrm{~V}$ and logic supply voltage can be in the range of 1.8 to $3.3 \mathrm{~V}$.
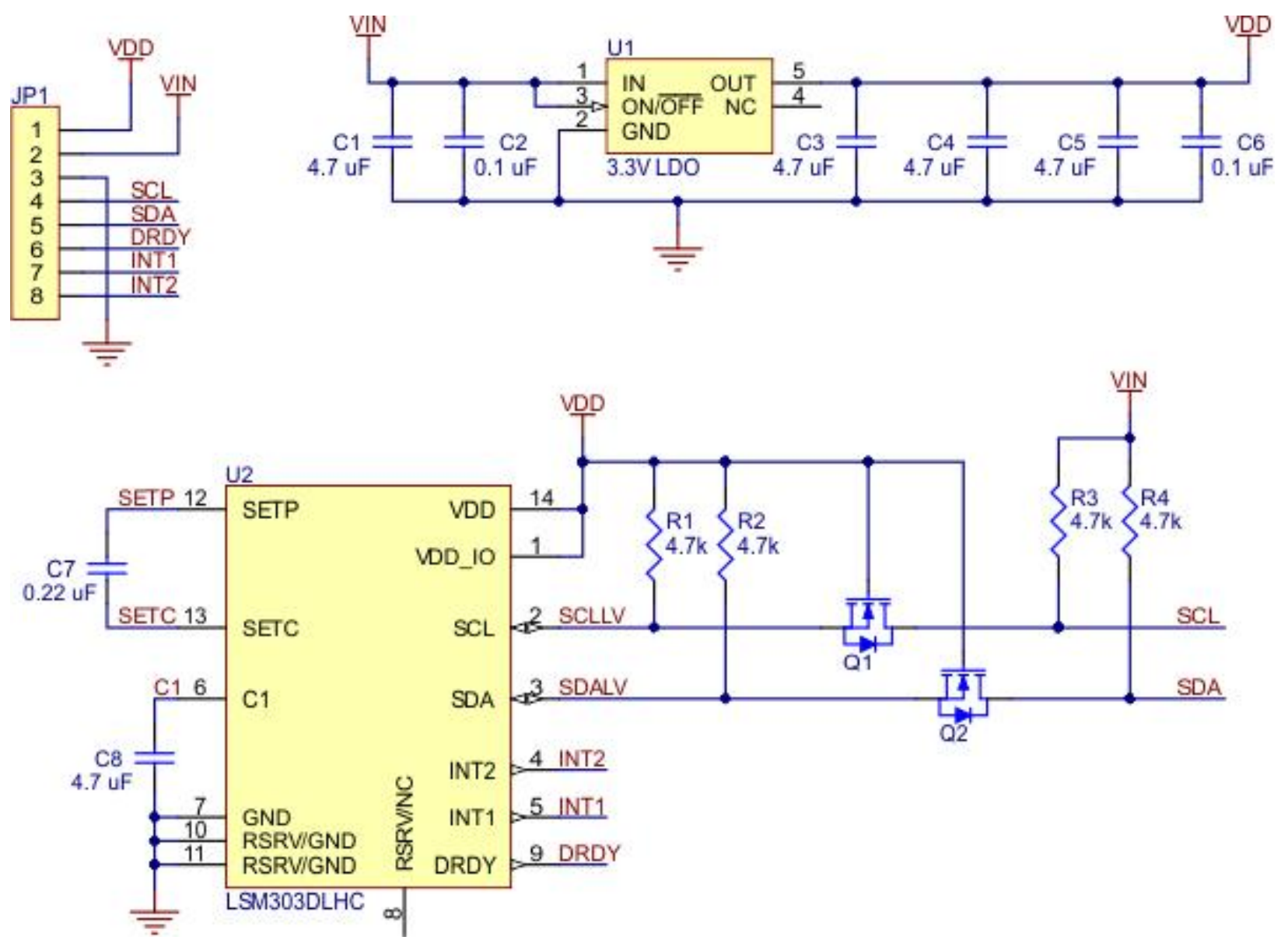

Fig. 9. Typical connection circuit

of the LSM303DLHC module to the microcontroller 
The LSM303DLHC module is connected through the $\mathrm{I}^{2} \mathrm{C}$ bus as a slave device. The microcontroller is a master device. In the digital compass design, the following has to be taken into account [9]:

- Reserved pins have to be connected according to the LSM303DLHC datasheet.

- Ceramic power source decoupling capacitors should be placed as close to the VDD (pin 6) as possible.

- The selected microcontroller must have a built-in $\mathrm{I}^{2} \mathrm{C}$ interface controller.

- Power and logic supplies should be adjustable and with small noises.

\section{Software of digital compass}

\section{Data acquisition from the accelerometer}

If the pin 4 SA0_A is connected to voltage supply, the 7-bit $\mathrm{I}^{2} \mathrm{C}$ slave address of the accelerometer will be $0 \times 19$. And otherwise if the pin 4 SA0_A is connected to ground, then the 7-bit $\mathrm{I}^{2} \mathrm{C}$ slave address of the accelerometer will be $0 \times 18$.

After powering on the LSM303DLHC module, the two registers CTRL_REG1_A (20h) and CTRL_REG4_A (23h) must be configured. For the normal operation mode (ODR=50 Hz), the value $0 \times 27$ is written to the register CTRL_REG1_A. To setup the continuous mode of the data update, the value $0 \times 40$ has to be written to the CTRL_REG4_A register [10]. In the following block-diagram, the algorithm of the accelerometer data acquisition is shown, Fig. 10:

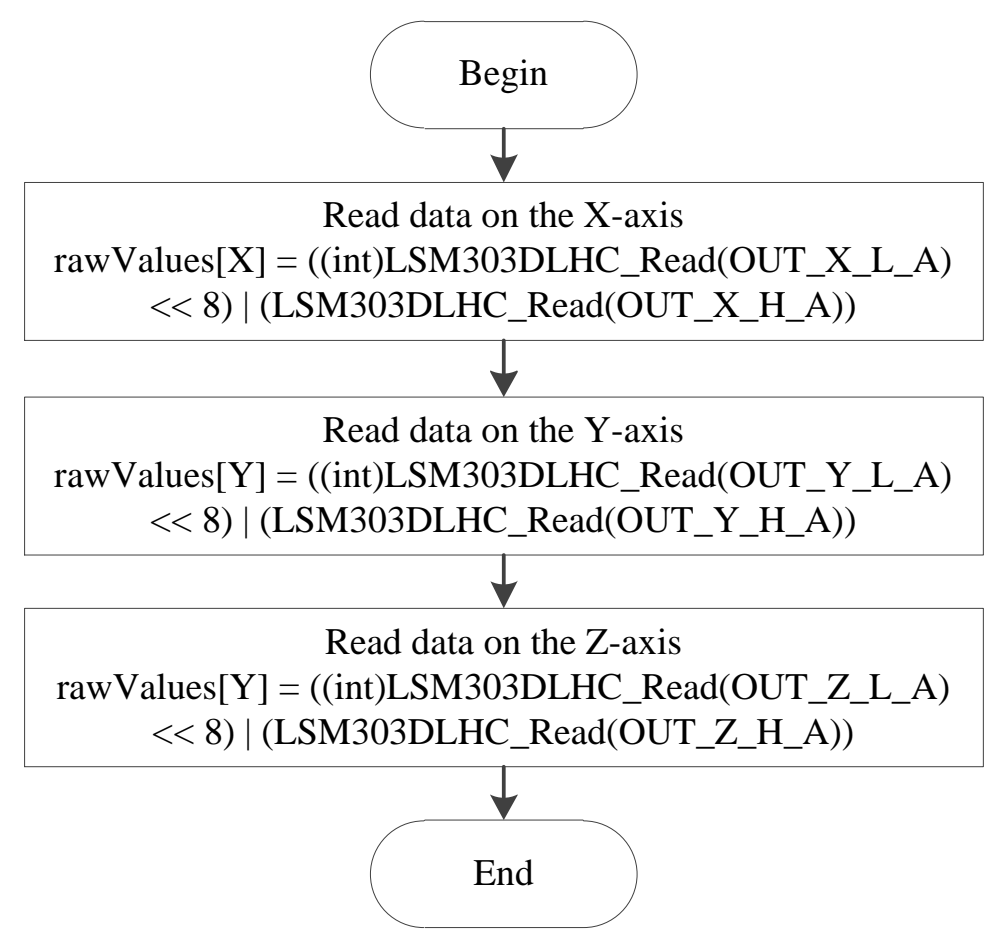

Fig. 10. The block-diagram

of the data acquisition from the accelerometer

\section{Data acquisition from the magnetometer LSM303DLHC}

The magnetometer has the 7-bit $\mathrm{I}^{2} \mathrm{C}$ slave address $0 \times 1 \mathrm{E}$. After powering on the LSM303DLHC module, the two registers CRA_REG_M (00h) and MR_REG_M (02h) have to be configured. In order to do this, the value $0 \times 14$ has to be written to the CRA_REG_M register for changing the data output rate from $15 \mathrm{~Hz}$ to $30 \mathrm{~Hz}$. The value $0 \times 00$ has be written to the register MR_REG_M to wake up the 
magnetometer for the normal operation mode [10]. The range \pm 1.3 gauss and data output rate (ODR) $30 \mathrm{~Hz}$ are sufficient for the digital compass.

The following block-diagram demonstrates the data acquisition algorithm from the magnetometer (Fig. 11):

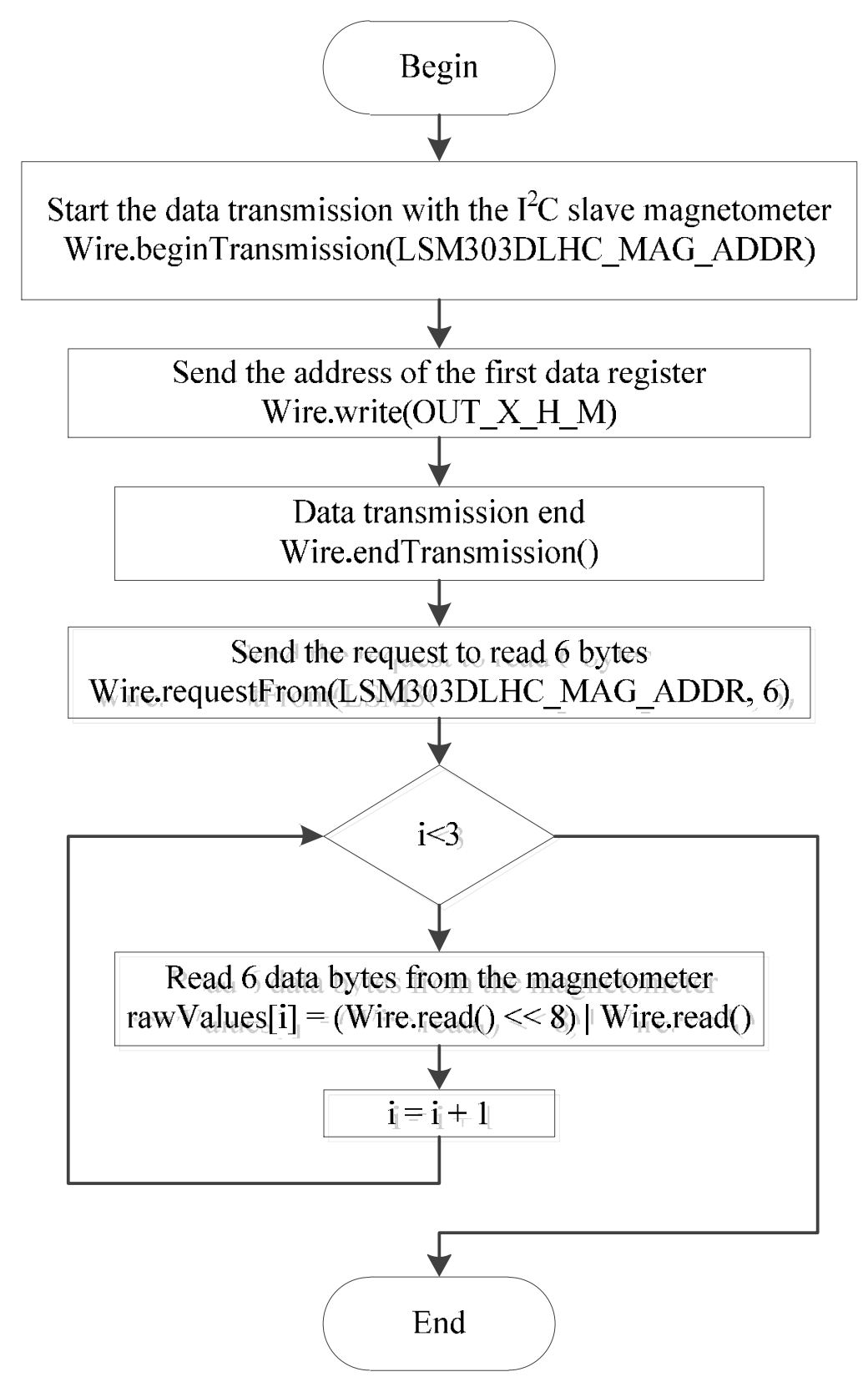

Fig. 11 The block-diagram of the data acquisition from the LSM303DLHC magnetometer

The pointer of the register address of the magnetometer is automatically updated. After reading the register MR_REG_M $(02 \mathrm{~h})$, the pointer will automatically increase by 1 to $03 \mathrm{~h}$ that is the address of the register OUT_X_H_M. After reading the register OUT_X_H_M, the address pointer will increase by 1 to the address of the register OUT_X_L_M. The embedded software of the digital compass is compiled into a hex file that is flashed into the ATmega328 microcontroller of the Arduino Uno platform. 


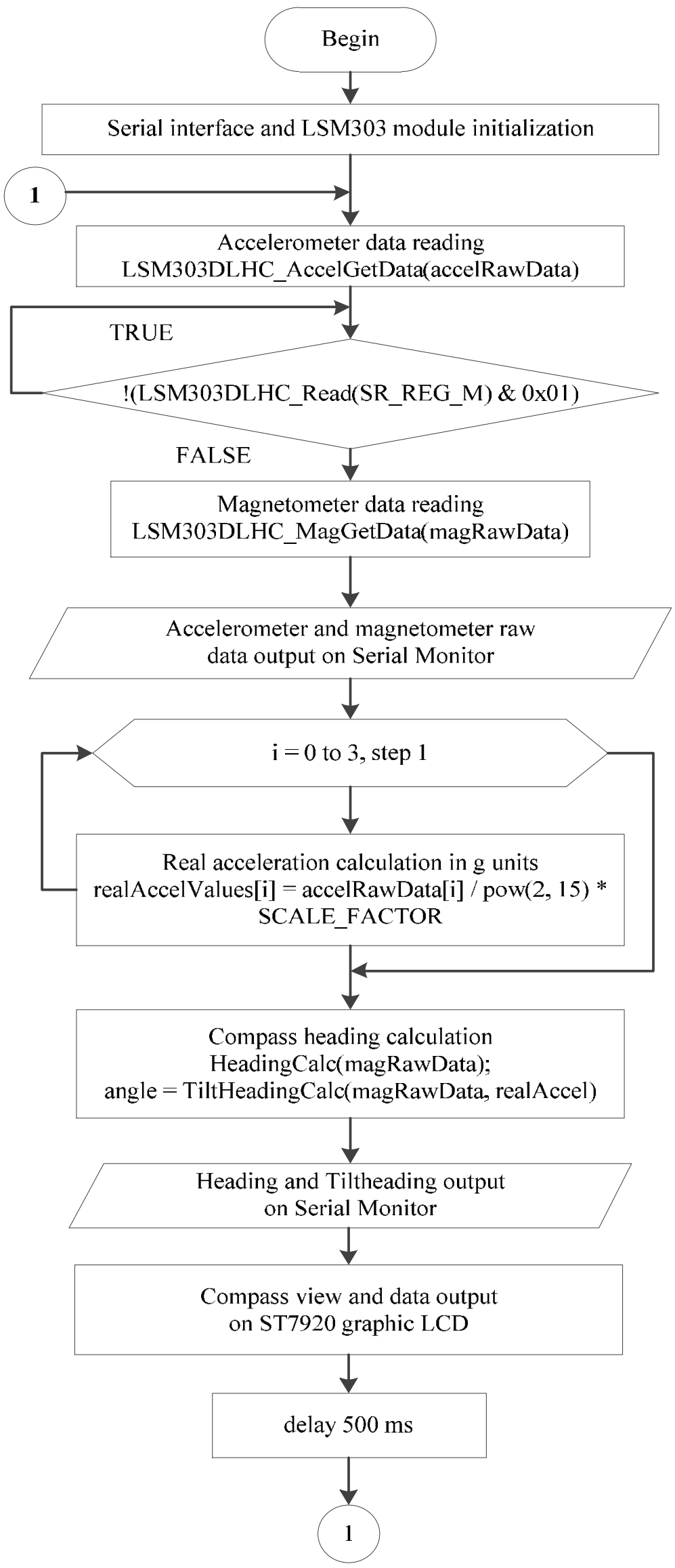

Fig. 12. Operation algorithm of the digital compass 


\section{Research Results and Their Analysis}

The research results are graphically presented in Fig. 13-16. The designed circuit of the digital device is simulated in Proteus (Fig. 13) and tested on the developed prototype (Fig. 14).

In Fig. 14-15 the raw data obtained on the x-, y-, z-axes from the accelerometer Ax, Ay, Az and magnetometer Mx, My, Mz has been shown. The graphical view of the compass heading and direction southeast (SE) is shown on the $128 \times 64$ graphic LCD ST7920 (Fig. 16).

Digital compass built on accelerometer+magnetomter LSM303DLHC

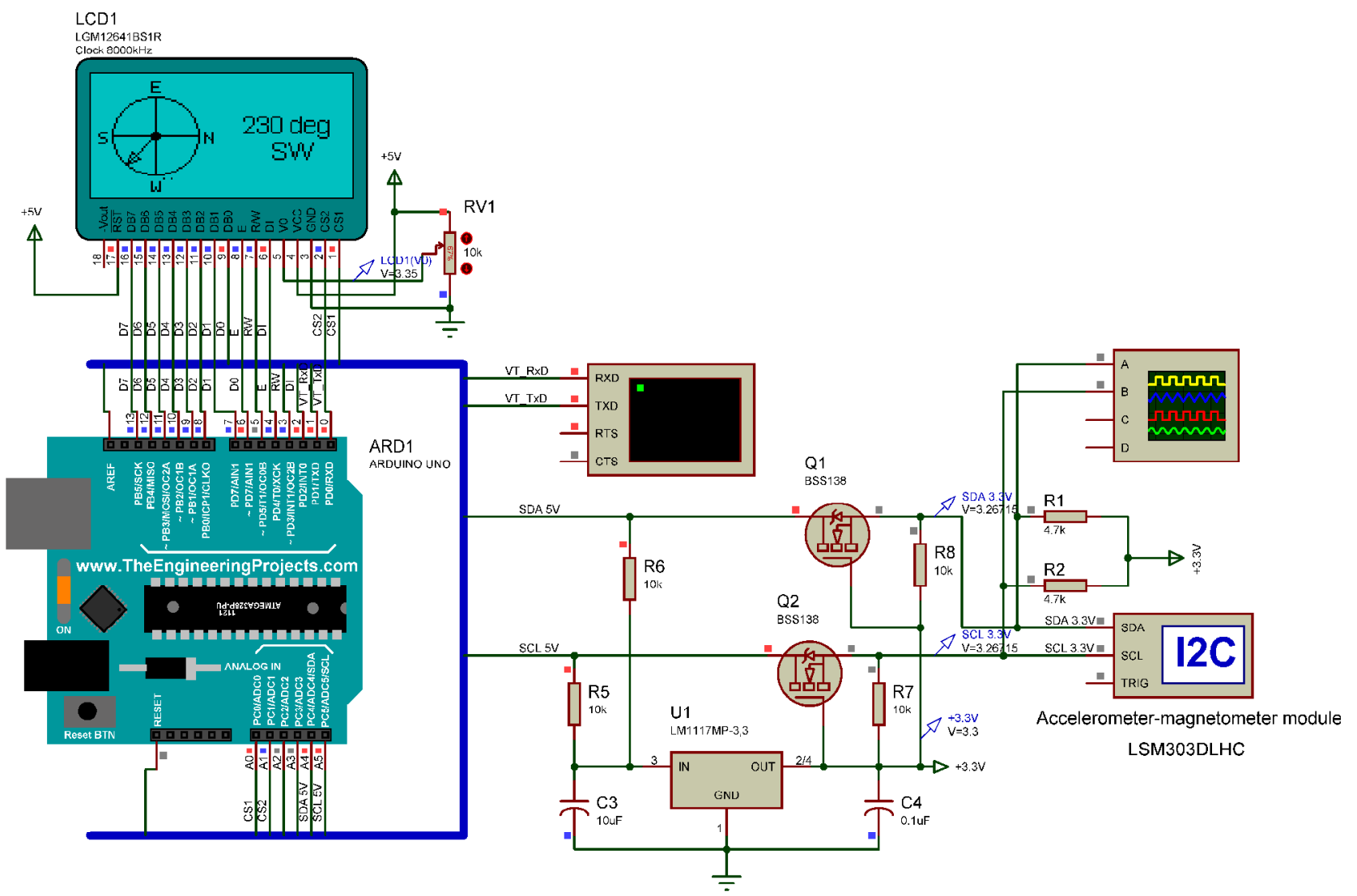

Fig. 13. Digital compass model developed in Proteus

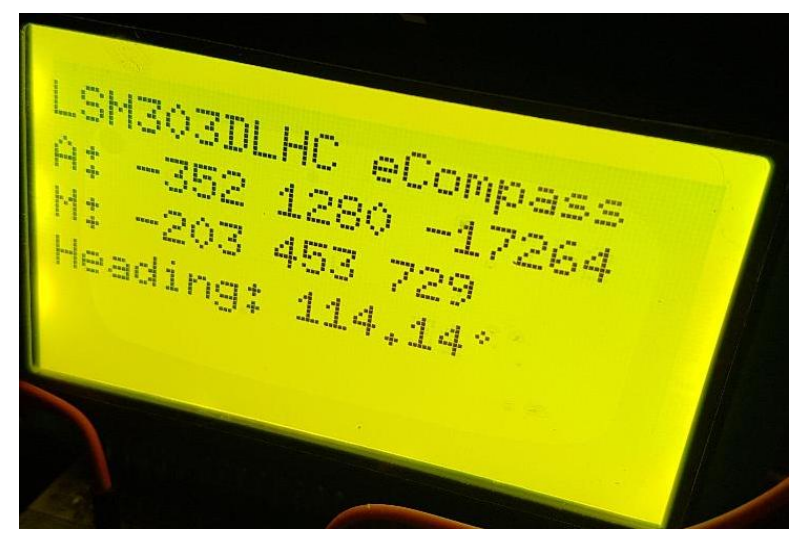

Fig. 14. Accelerometer and magnetometer raw data displayed on the graphic LCD ST7920 


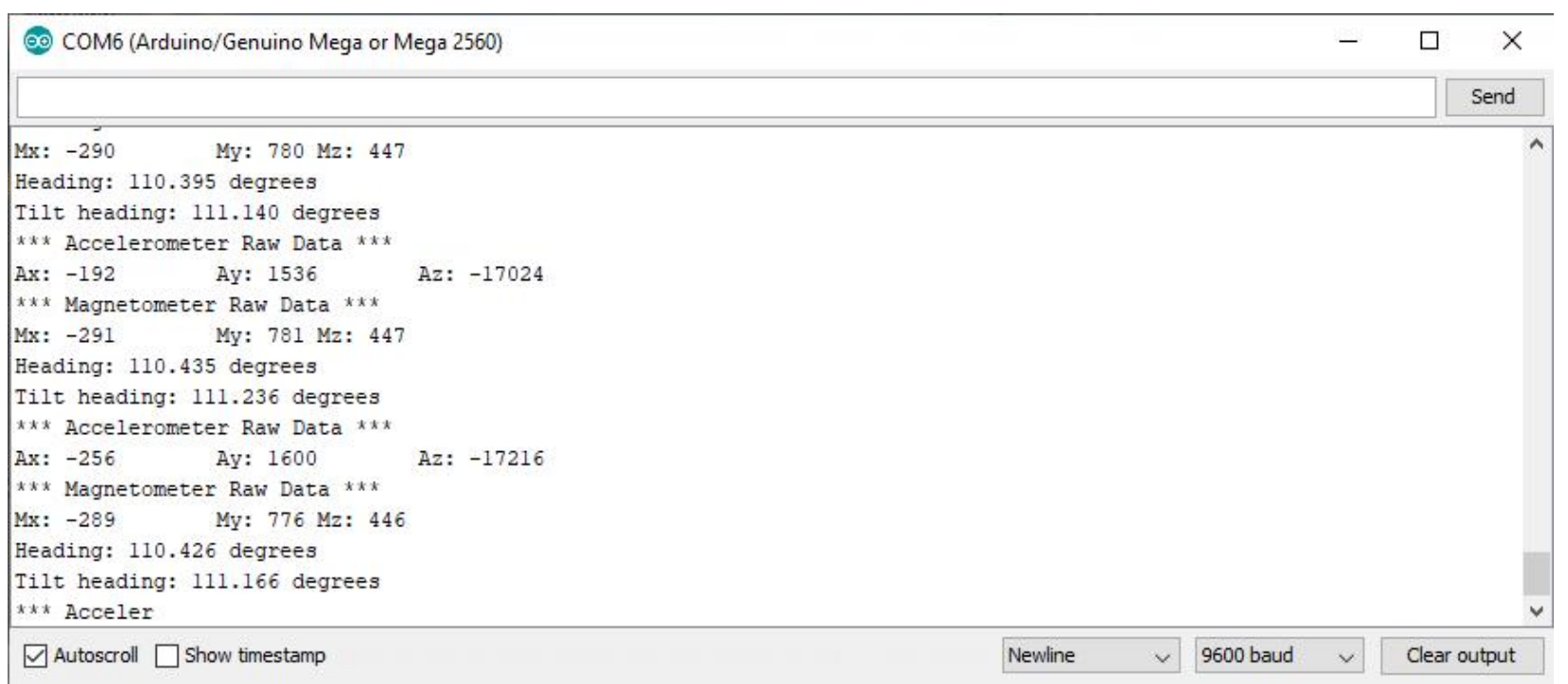

Fig. 15. Accelerometer and magnetometer raw data output on the serial monitor

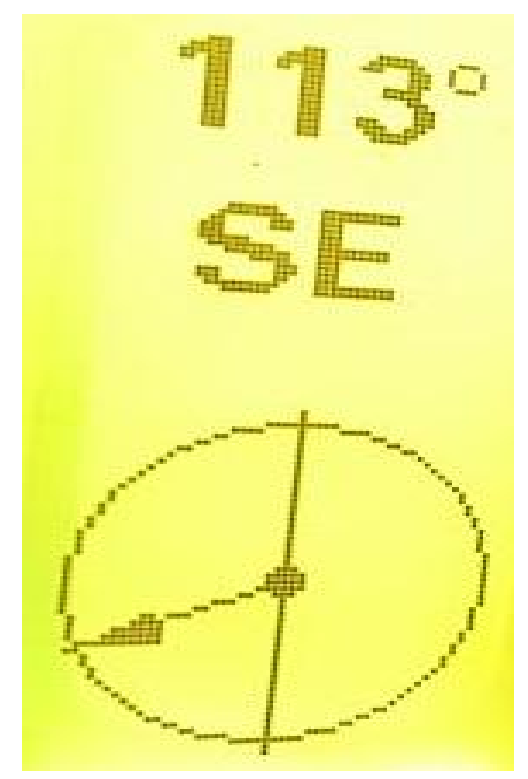

Fig. 16. Graphical indication of the compass heading on the $128 \times 64$ graphic LCD $S T 7920$

\section{Conclusion}

The digital compass based on the Arduino Uno platform using the MEMS module (accelerometermagnetometer) LSM303DLHC has been developed. The structure of the digital compass hardware has been developed. The algorithms for accelerometer and magnetometer calibration, for calculating roll and pitch angles have been developed. The algorithm of compass heading calculation without tilt compensation and with tilt compensation has been developed. The embedded software of the digital compass has been developed. The model and prototype of the digital compass have been created. The research and result analysis of the digital compass operation have been conducted.

1. Electronic resource: https://www.st.com/content/st_com/en/products/mems-and-sensors/ecompasses.html

2. Electronic resource: https://www.memsic.com/magnetic-sensors/ 
3. Compass." [Online]. Available: http://en.wikipedia.org/wiki/Compass[2] "Lodestone". [Online] .Available: http://en.wikipedia.org/wiki/Lodestone

4. Electronic resource: https://en.wikipedia.org/wiki/Earth\%27s_magnetic_field

5. Electronic resource: https://www.livescience.com/32732-how-does-a-compass-work.html

6. Grygorenko V., "Cypress application note - magnetic compass with tilt compensation-an2272". [Online].Available: http://www.cypress.com/?docID=221.

7. Application note AN4248: "Implementing a Tilt-Compensated eCompass using Accelerometer and Mag-netometer Sensors”, 2012, http://cache.freescale.com/files/sensors/doc/app_note/AN4248.pdf.

8. Application note AN3192: "Using LSM303DLH for a tilt compensated electronic compass" https://www.pololu.com/file/OJ434/LSM303DLH-compass-app-note.pdf.

9. Electronic resource: https://www.st.com/en/mems-and-sensors/lsm303dlhc.html

10. Electronic resource: https://www.st.com/resource/en/datasheet/DM00027543.pdf

А. Головатий, В. Теслюк, К. К. Колесник, В. В. Табала Національний університет "Львівська політехніка"

\section{РОЗРОБКА ЦИФРОВОГО КОМПАСУ HA AVR МІКРОКОНТРОЛЕРІ ТА МЕМS - МОДУЛІ АКСЕЛЕРОМЕТРА - MAГНITOMETPA LSM303DLHC}

(C) Головатий А., Теслюк В., Колесник К., Табала В., 2019

У роботі розроблено цифровий компас на MК AVR з використанням МЕМС - модуля акселерометра - магнітометра LSM303DLHC. Розроблено структуру та спроектовано апаратне забезпечення цифрового компасу. Описано алгоритми калібрування акселерометра та магнітометра LSM303DLHC. Описано алгоритм обчислення кутів нахилу (кутів тангажу і крену) цифрового компасу. Описано алгоритм визначення курсу (напрямку) компаса без компенсації і 3 компенсацісю нахилу. Розроблено алгоритм роботи та програмне забезпечення цифрового компасу. Створено модель та макет цифрового компасу. Проведено моделювання і тестування розробленого цифрового компасу.

Ключові слова: цифровий компас, апаратно-програмна платформа Arduino Uno R3, MK ATmega328P-PU, акселерометр, магнітометр, МЕМС модуль LSM303DLHC, CAПP Proteus Design Suite, мова програмування C, середовище програмування Arduino IDE для МК платформи Arduino, вбудоване програмне забезпечення. 